\title{
Improvement of Nemadectin Production by Overexpressing the Regulatory Gene nemR and Nemadectin Biosynthetic Gene Cluster in Streptomyces Cyaneogriseus
}

\author{
Yuan-Jie Wu ${ }^{1} \quad$ Song-Bai Yang ${ }^{1} \quad$ Zheng-Yu Zhang ${ }^{1}$
}

Address for correspondence Shao-Xin Chen, PhD, State Key Laboratory of New Drug and Pharmaceutical Process, Shanghai Institute of Pharmaceutical Industry, China State Institute of Pharmaceutical Industry, Shanghai 201203, People's Republic of China (e-mail: sxzlb@263.net).

\begin{abstract}
Keywords

- nemadectin

- overexpression

- biosynthesis gene cluster

- Streptomyces cyaneogriseus
\end{abstract}

Nemadectin, a 16-member macrocyclic lactone antiparasitic antibiotic, is produced by Streptomyces cyaneogriseus subspecies noncyanogenus. Moxidectin, a $\mathrm{C}-23$ oximate derivative of nemadectin, is widely used as a pesticide due to its broad-spectrum, highly efficient, and safe anthelmintic activity. NemR, a LAL family regulator, is encoded by nemR and is involved in nemadectin biosynthesis in S. cyaneogriseus. In this report, gene disruption and complementation experiments showed that nemR plays a positive role in the biosynthesis of nemadectin. The transcription level of nemadectin biosynthetic genes in the nemR knockout strain was significantly decreased compared with those in the wild-type strain MOX-101. However, overexpression of nemR under the control of native or strong constitutive promoters resulted in the opposite, increasing the production of nemadectin by 56.5 or $73.5 \%$, respectively, when compared with MOX-101. In addition, the gene cluster of nemadectin biosynthesis was further cloned and overexpressed using a CRISPR method, which significantly increase nemadectin yield by $108.6 \%$ (509 mg/L) when compared with MOX-101.

\section{Introduction}

Nemadectin produced by Streptomyces cyaneogriseus subspecies noncyanogenus is a 16-member macrocyclic lactone antiparasitic antibiotic with broad-spectrum endectocidal and nematocidal activity. ${ }^{1,2}$ The structure of nemadectin is similar to those of milbemycin, avermectin, and meilingmycin. ${ }^{3-5}$ Moxidectin, a C-23 oximate derivative of nemadectin (-Fig. 1), showed stronger insecticidal activity than nemadectin. ${ }^{6}$ Moxidectin has many advantages over avermectin, ivermectin, and other insect-repellent antibiotics, such as low toxicity, high efficiency, and a broader spectrum. ${ }^{7-10}$ In 2018, the U.S. Food and Drug Administration approved moxidectin for the treatment of onchocerciasis (river blindness) in patients aged 12 years and older.

The preliminary research on nemadectin was mainly focused on strain breeding and optimization of fermentation technology. In 2009, the yield of the strain was increased to $172 \mathrm{mg} / \mathrm{L}$ by ultraviolet (UV) mutagenesis combined with the screening of precursor resistance. ${ }^{11}$ In 2014, the fermentation process of nemadectin was optimized by investigating various factors, such as time, temperature, and dissolved oxygen. ${ }^{12}$ In 2015, Li et al reported a method for improving nemadectin production by screening a mutant strain DC18-01 and adding the precursor sodium acetate for one or more times in the middle and later stages of fermentation. ${ }^{13}$ Although the received

July 24,2020

accepted

December 17, 2020

published online

January 22, 2021
DOI https://doi.org/

10.1055/s-0040-1722746.

ISSN 2628-5088. (c) 2021. The Author(s).

This is an open access article published by Thieme under the terms of the Creative Commons Attribution License, permitting unrestricted use, distribution, and reproduction so long as the original work is properly cited. (https://creativecommons.org/licenses/by/4.0/)

Georg Thieme Verlag KG, Rüdigerstraße 14, 70469 Stuttgart, Germany 


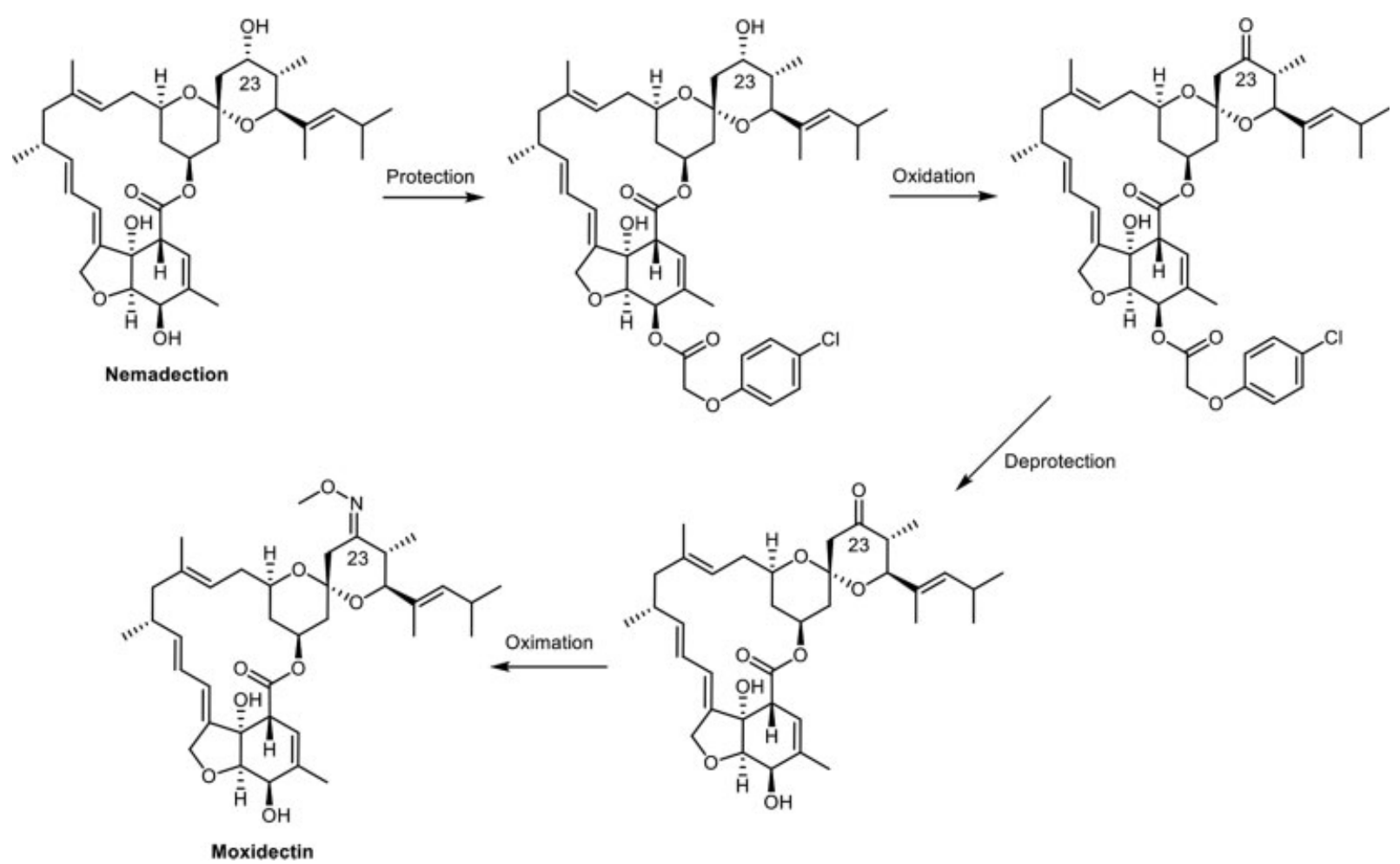

Fig. 1 The semi-chemical synthesis of moxidectin.

biosynthetic gene cluster of nemadectin has been identified, and its complete sequence is also available,$^{14}$ the study on genetic modification to nemadectin-producing strain remains largely unknown. In 2019, the function of the gene nemR was characterized, which is a positive regulatory gene encoding a LAL family transcriptional regulator within the nemadectin biosynthesis gene cluster of the strain NMWT1 $1{ }^{15}$ The low fermentation yield of nemadectin resulted in the high production cost of moxidectin. In this study, the LAL family regulatory gene nemR, which serves as an activator for nemadectin biosynthesis, was confirmed and was employed to improve nemadectin production by overexpression of nemR in the strain MOX-101. The nemadectin biosynthesis gene cluster in MOX-101 was successfully cloned and used to increase nemadectin production by duplicating of the biosynthesis gene cluster in S. cyaneogriseus.

\section{Materials and Methods}

\section{Strains, Plasmids, and Primers}

Strains and plasmids used in this study are listed in - Table $\mathbf{1}$. Primers are listed in - Table $\mathbf{2}$.

\section{Construction of Gene Deletion, Complementation, and Overexpression Strains}

The construction process for the $\Delta$ nem $\mathrm{R}$ mutant with deletion of nem $\mathrm{R}$ is as follows. A 1.5-kb fragment upstream of the nem $\mathrm{R}$ start codon and a 1.5 -kb fragment downstream of the nem $\mathrm{R}$ stop codon were amplified from MOX-101 genomic DNA (gDNA) using the primer pairs nemR-up-F/R and nemR-down-F/R, respectively. Two polymerase chain reaction (PCR) fragments were spliced together through overlapping PCR using the primers nemR-up-F and nemRdown-R. The resulting product was cloned into a 3.1-kb DNA fragment that was amplified from pSET152 using the primers pSET152-F/R to obtain the nemR-deleted plasmid $\mathrm{p} \Delta$ nem $\mathrm{R}$ using the seamless ligation method (ClonExpress II One Step Cloning kit) (-Fig. 2A). The plasmid p $\mathbf{\Delta}$ nem $\mathrm{R}$ was transferred from S17-1 into MOX-101 by intergeneric conjugation. After selecting single-crossover recombinant strains and double-crossover recombinant strains, the resulting mutant with deletion of nemR was named $\Delta$ nemR.

The nem $\mathrm{R}$ complementation strain $\Delta$ nem $\mathrm{R} / \mathrm{pnem} \mathrm{R}$ was constructed as follows. Using the gDNA of MOX-101 as the template, a 3.7-kb DNA fragment containing the gene nem $\mathrm{R}$ and its promoter region was amplified by PCR with the primers nemR-N-F/R. The PCR product was inserted into the $\mathrm{XbaI} / \mathrm{BamHI}$ site of pSET152 to generate the integrative plasmid pnemR ( - Fig. 2B). The sequence of nemR in pnem $\mathrm{R}$ was confirmed by DNA sequencing. After conjugal transfer, pnem $\mathrm{R}$ was transferred into the $\Delta$ nem $\mathrm{R}$ strain to obtain the $n e m \mathrm{R}$ complementation strain $\Delta$ nemR/pnemR. As a control, the control plasmid pSET152 was transferred into MOX-101 to generate the strain MOX-101/pSET152.

The gene nem $\mathrm{R}$ was amplified from MOX-101 gDNA using the primers nemR-Q-F/R and cloned into the NdeI/AscI sites of PSET152 to generate the overexpression plasmid pnemRermEp*, in which nem $\mathrm{R}$ is driven by the strong constitutive promoter ermEp* (- Fig. $\mathbf{2 C}$ ). Next, pnemR bearing the native promoter and pnemR-ermEp* were transferred into MOX101 to obtain overexpression strains MOX-101/pnemR and MOX-101/pnemR-ermEp*. 
Table 1 Strains and plasmids used in this study

\begin{tabular}{|c|c|c|}
\hline Strains or plasmids & Relevant characteristic & $\begin{array}{l}\text { Reference } \\
\text { or source }\end{array}$ \\
\hline \multicolumn{3}{|l|}{ Strains } \\
\hline \multicolumn{3}{|l|}{ E. coli } \\
\hline $\mathrm{DH} 5 \alpha$ & 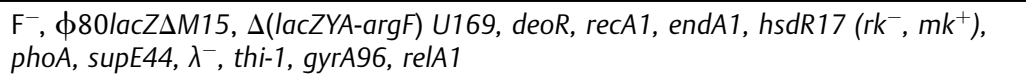 & CWBIO \\
\hline S17-1 & $\begin{array}{l}\text { supE44, } \Delta \text { lacU169 (FlacZAM15), recA1, endA1, hsdR17, thi-1, gyrA96, relA1, par } \\
\text { phage lysogenic }\end{array}$ & Gibco-BRL \\
\hline EPI300 & 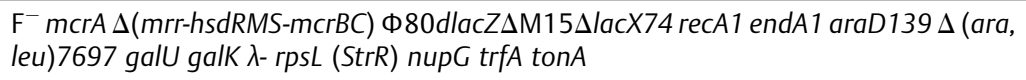 & Epicentre \\
\hline \multicolumn{3}{|l|}{ S. cerevisiae } \\
\hline VL6-48 & MATa his3-D200 trp1-D1 ura3-52 ys2ade2-101 met14 psi+ cir0 & ATCC MYA-366 \\
\hline \multicolumn{3}{|l|}{ S. cyaneogriseus } \\
\hline MOX-101 & Nemadectin producer & This laboratory \\
\hline$\Delta$ nemR & MOX-101 with deleted nemR & This study \\
\hline$\Delta$ nemR/pnemR & $\Delta$ nemR mutant with pnemR & This study \\
\hline MOX-101/pSET152 & MOX-101 strain with pSET152 & This study \\
\hline MOX-101/pnemR & MOX-101 strain with pnemR & This study \\
\hline MOX-101/pnemR-ermEp* & MOX-101 strain with pnemR-ermEp* & This study \\
\hline MOX-101/pCL01 & MOX-101 strain with pCL01 & This study \\
\hline MOX-101/pCL-MOX & MOX-101 strain with $\mathrm{pCL}-\mathrm{MOX}$ & This study \\
\hline \multicolumn{3}{|l|}{ Plasmids } \\
\hline pSET152 & E. coli replicon, Streptomyces $\emptyset \mathrm{C} 31$ attachment site, $\mathrm{Apr}^{\mathrm{R}}$ & This laboratory \\
\hline pKCCas9(tipAp) & pKC1139 with the scocas9 gene under the control of the inducible promoter tipAp & 16 \\
\hline pCL01 & $\begin{array}{l}\text { Large gene cluster capture vector: derived from pCC1BAC containing a single copy } \\
\text { F-factor replicon and a high-copy origin of replication, } \Phi C 31 \text { int/attP, aac(3)IV, oriT } \\
\text { RK2, ARSH4/CEN6, and TRP1 }\end{array}$ & 17 \\
\hline $\mathrm{p} \Delta \mathrm{nemR}$ & pSET152 with the upstream and downstream DNA fragments of nemR & This study \\
\hline pnemR & pSET152 with the expression of nemR under the control of native promoter & This study \\
\hline pnemR-ermEp* & pSET152 with the expression of nemR under the control of strong promoter ermEp* & This study \\
\hline pCL-M1 & $\begin{array}{l}\text { pCL01 with the upstream and downstream DNA fragments of the gene cluster } \\
\text { MOX1 }\end{array}$ & This study \\
\hline $\mathrm{pCL}-\mathrm{M} 2$ & $\begin{array}{l}\text { pCL01 with the upstream and downstream DNA fragments of the gene cluster } \\
\text { MOX2 }\end{array}$ & This study \\
\hline $\mathrm{pCL}-\mathrm{M}$ & $\begin{array}{l}\text { pCL01 with the upstream and downstream DNA fragments of the gene cluster } \\
\text { MOX }\end{array}$ & This study \\
\hline pCL-MOX1 & pCL-M1 with the biosynthetic gene cluster MOX1 & This study \\
\hline pCL-MOX2 & $\mathrm{pCL}-\mathrm{M} 2$ with the biosynthetic gene cluster MOX2 & This study \\
\hline pCL-MOX & pCL-M with the biosynthetic gene cluster MOX & This study \\
\hline
\end{tabular}

\section{Construction of Nemadectin Biosynthetic Gene Cluster Overexpression Strain}

Cloning nemadectin biosynthetic gene cluster was conducted following the CRISPR-TAR method reported previously. ${ }^{18}$ The nemadectin biosynthetic gene cluster MOX was divided into two parts, namely, MOX1 (50 kb) and MOX2 (41.7 kb). MOX1 and MOX2 were cloned respectively, and then two modules were spliced to generate the complete nemadectin biosynthetic gene cluster.

CRISPR-digested gDNA was prepared as follows. First, four gRNA minigenes encoding gRNA were amplified from the plasmid pKCCas9 (tipAp) by the primers gRNA-MOX1-
up-F/gRNA-R, gRNA-MOX1-down-F/gRNA-R, gRNA-MOX2up-F/gRNA-R, and gRNA-MOX2-down-F/gRNA-R designed at each end of MOX1 and MOX2 gene clusters, respectively. Next, the resulting products were transcribed in vitro by MEGAScriptTMT7 Kit (Thermo Fisher Scientific, China) to obtain gRNAs used to guide Cas9 nuclease to cleave the gDNA of MOX-101. These gRNAs were purified using MEGAClear Kit (Thermo Fisher Scientific, China). Then, the gDNA isolated by the phenol-chloroform method was digested in vitro overnight with the help of the Cas9 nuclease and gRNAs.

Plasmids pCL-M1, pCL-M2, and pCL-M were constructed to capture MOX1, MOX2, and MOX gene clusters, respectively. 
e154 Roles of nemR and Nemadectin Biosynthetic Gene Cluster in Regulating Nemadectin Production Wu et al.

Table 2 Primers used in this study

\begin{tabular}{|c|c|}
\hline Primers & Sequences $\left(5^{\prime} \rightarrow 3^{\prime}\right)^{a}$ \\
\hline nemR-up-F & AAAGATCCGTCGACCTGCAGAAGCTTGCCCTACGCCATGACCAAGG \\
\hline nemR-up-R & GTTCAGACCGGGCTTTCGATAATCTCCCTCTGACTCCCTCCCC \\
\hline nemR-down-F & ATCGAAAGCCCGGTCTGAACG \\
\hline nemR-down-F & TCGCGCGCGGCCGCGGATCCTCTAGAGGCAGCAGGATCATCGGTGG \\
\hline pSET152- F & GACTCTAGAGGATCCGCGGC \\
\hline pSET152- R & AAGCTTCTGCAGGTCGACGGA \\
\hline nemR-N-F & AGCTTGGGCTGCAGGTCGACTCTAGAAGCCAGAGGGATTCGGTCTC \\
\hline nemR-N-R & TCGATATCGCGCGCGGCCGCGGATCCTCAGACCGGGCTTTCGATGT \\
\hline nemR-Q-F & aaccactccacaggaggacccatatgATGCGGGGCGTTTCCCCTTC \\
\hline nemR-Q-R & TGGAAAGACGACAAAACTTTGGCGCGCCTCAGACCGGGCTTTCGATGTGC \\
\hline nemA1-2A2-F & tggacagggacgacagcgcc \\
\hline nemA1-2A2-R & ACCGGAGCTGACGAGGTCCC \\
\hline nemA2C-F & ggctcttgacgtcgatcccga \\
\hline nemA2C-R & TGTCGAGGACCCTTCGGTGG \\
\hline nemA3E-F & gggtggcgaggtagacggtg \\
\hline nemA3E-R & CCAGATCGCCACGGTCCTCG \\
\hline nemA4A3-F & ccagcagcaagcgctgctgg \\
\hline nemA4A3-R & AGCCGCACACCGTCCTCGAA \\
\hline nemG-F & ATGCCCGACCTTTGCGAGACC \\
\hline nemG-R & CGGTCCTGTCGTCCGGGGTAC \\
\hline nemF-F & CAGGACGGCCTTGGTCACGG \\
\hline nemF-R & AGAGCTCATCCACGGCGTCGTC \\
\hline nemA1-2-F & GTGGGTCACCGCCGATCTGC \\
\hline nemA1-2-R & ACAGCCCGTCCAGATCCCACC \\
\hline nemA3-F & CGAGAAGCTCGTCGAGGCGCT \\
\hline nemA3-R & AGCCACCAGCCCCCACAACTC \\
\hline nemA4-F & TGCTCCGCGAGTACCTCAAGCG \\
\hline nemA4-R & GGGAAGTCGCCGATCGCGTC \\
\hline gRNA-MOX1-up-F & gactgacactgaTAATACGACTCACTATAggagaggacgagccggtagcGTTTTAGAGCTAGAAATA \\
\hline gRNA-MOX1-down-F & gactgacactgaTAATACGACTCACTATAggtcacgacgccgtggcagcGTTTTAGAGCTAGAAATA \\
\hline gRNA-MOX2-up-F & gactgacactgaTAATACGACTCACTATAggcgctcggcgcggtgtgccGTTTTAGAGCTAGAAATA \\
\hline gRNA-MOX2-down-F & gactgacactgaTAATACGACTCACTATAggcgtcccgcgtccccgtgcGTTTTAGAGCTAGAAATA \\
\hline gRNA-R & CTCAAAAAAAGCACCGACTCGG \\
\hline MOX1-up-F & agtgaattgtaatacgactcactatagggcatttaaatGAGGACGTTCACCCGTGGTTC \\
\hline MOX1-up-R & CGACTCCCGAGGACAAGATGG \\
\hline MOX1-down-F & CagcgccatcttgtcctcgggagtcgGTTTAAACACGTCCGAGTTGCGTGAGCG \\
\hline MOX1-down-R & gcacgtgatgaaaaggacccaggtggcacACGTGCGTGTTGGAGACCAG \\
\hline MOX2-up-F & agtgaattgtaatacgactcactatagggcatttaaatATCTGGGTGCCCGCCTGCTT \\
\hline MOX2-up-R & GCCGTATAGCCGCCCATCA \\
\hline MOX2-down-F & cagcgccatcttgtcctcgggagtcgGTTTAAACGCGTTGGTGGTGACCGTGAG \\
\hline MOX2-down-R & gcacgtgatgaaaaggacccaggtggcacCGACCTCAACCGCTTCTACACC \\
\hline MOX-down-F & cagcgccatcttgtcctcgggagtcgGTTTAAACGCGTTGGTGGTGACCGTGAG \\
\hline
\end{tabular}

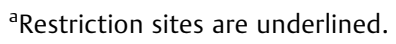


A

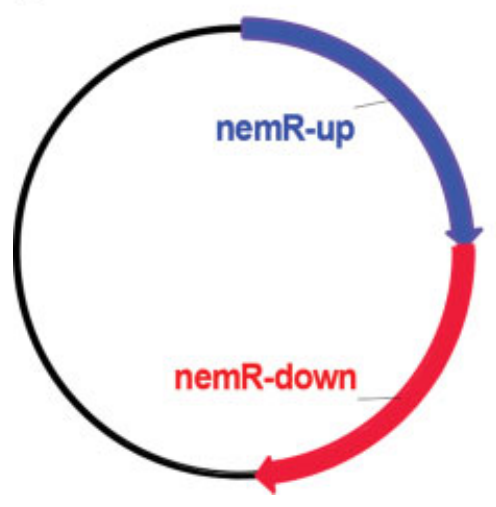

B

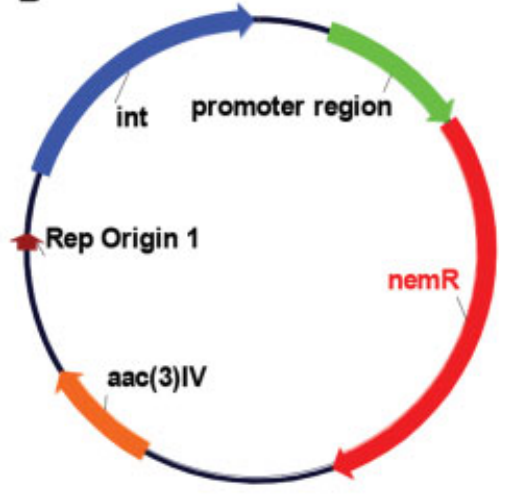

C

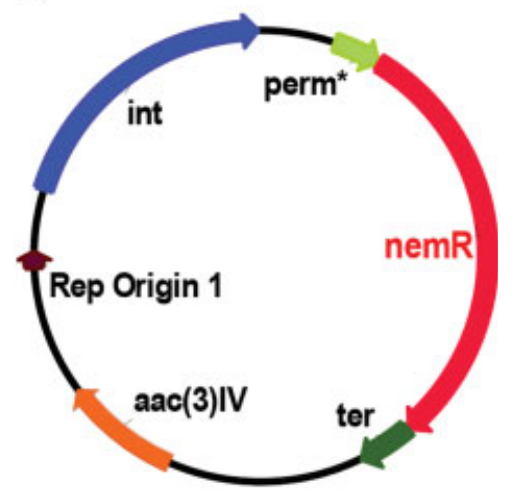

Fig. 2 Plasmid maps of p $\Delta$ nemR (A), pnemR (B), and pnemR-ermEp* (C).

Using the gDNA of MOX-101 as the template, six homologous arms, MOX1-up, MOX1-down, MOX2-up, MOX2-down, MOX-up, and MOX-down, were amplified with the primers MOX1-up-F/R, MOX1-down-F/R, MOX2-up-F/R, MOX2down-F/R, MOX1-up-F/R, and MOX-down-F/MOX2-down-R, respectively. Then, MOX1-up and MOX1-down, MOX2-up and MOX2-down, and MOX1-up and MOX2-down were spliced together by overlapping PCR, respectively. The resulting fragments were cloned into the EcoRI/Swal site of the plasmid pLC01 by homologous recombination to generate the capture plasmids pCL-M1, pCL-M2, and pCL-M, respectively. Capture plasmids were digested with restriction enzyme PmeI to obtain linearized capture plasmids.

Digested genome DNAs and linearized capture plasmids were introduced into Saccharomyces cerevisiae VL6-48 to clone the biosynthetic gene cluster MOX1 and MOX2. After verifying transformants, positive plasmids pCL-MOX1 and pCL-MOX2 were transferred into Escherichia coli EPI300 for enrichment. Positive plasmids PCL-MOX1 and pCL-MOX2 were collected by E.Z.N.A.BAC/PAC DNA (Omega Bio-Tek, China) and digested with restriction enzyme Swal to obtain gene clusters MOX1 and MOX2, respectively. The obtained gene clusters and the linearized capture plasmid pCL-M were introduced into the yeast to clone the biosynthetic gene cluster MOX. After confirming the transformant, the positive plasmid pCL-MOX was transferred into E. coli EPI300 for enrichment. Plasmids pCL01, pCL-MOX1, pCL-MOX2, and pCL-MOX were digested with the restriction enzyme KpnI to verify if the captured gene clusters were correct. The correct plasmid pCL-MOX was transferred from S17-1 into MOX-101 to generate the engineering strain MOX-101/pCL-MOX, in which the nemadectin biosynthesis gene cluster was overexpressed. As a control, the control plasmid pCL01 was transferred into MOX-101 to generate the strain MOX-101/pCL01.

\section{Culture of Streptomyces Cyaneogriseus}

For nemadectin production, the parental strain MOX-101 and mutant strains were first grown on solid medium $(\mathrm{g} / \mathrm{L})$ : 4.0 glucose, 1.3 maltose, 4.0 yeast extract, 5.0 starch, 5.0 soybean meal, 5.0 dextrin, $1.0 \mathrm{KNO}_{3}, 0.5 \mathrm{~K}_{2} \mathrm{HPO}_{4}, 0.5 \mathrm{NaCl}, 0.5$ $\mathrm{MgSO}_{4}, 0.1 \mathrm{CaCO}_{3}$, and 20.0 agar at $\mathrm{pH} 6.8$ to 7.0 and $28^{\circ} \mathrm{C}$ for 10 to 12 days. Then, the mycelium was inoculated into a
250-mL flask with $20 \mathrm{~mL}$ seed medium (g/L): 10.0 glucose, 10.0 yeast meal, 5.0 yeast extract, 15.0 soybean meal, 20.0 dextrin, $1.0 \mathrm{~K}_{2} \mathrm{HPO}_{4}, 1.0 \mathrm{MgSO}_{4}$, and $4.0 \mathrm{CaCO}_{3}$, and the culture was grown at $\mathrm{pH} 7.0$ to $7.2,28^{\circ} \mathrm{C}$, and $200 \mathrm{rpm}$. After 24 to 28 hours, $10 \%$ seed culture was transferred into a 250 -mL flask with $25 \mathrm{~mL}$ fermentation medium $(\mathrm{g} / \mathrm{L})$ : 80.0 glucose, 5.0 yeast meal, 35.0 lactose, 27.5 soybean meal, 2.5 corn meal, $1.0 \mathrm{MgSO}_{4}$, and $4.0 \mathrm{CaCO}_{3}$, and the culture was grown at $\mathrm{pH} 7.0$ to $7.2,28^{\circ} \mathrm{C}$, and $200 \mathrm{rpm}$ for 8 days.

\section{Analytical Method}

To analyze nemadectin yield, $1.0 \mathrm{~mL}$ culture broth (collected for 4,6 , and 8 days) was extracted with $3.0 \mathrm{~mL}$ methanol for 30 minutes and centrifuged at $10,000 \times \mathrm{g}$ for 5 minutes. The supernatant was analyzed by high-pressure liquid chromatography (HPLC) with a Hypersil C18 column $(5 \mu \mathrm{m}, 4.6 \mathrm{~mm}$ $\times 150 \mathrm{~mm}$ ). The column was maintained at $30^{\circ} \mathrm{C}$ with mobile phases of methanol:water (85:15 [ $\mathrm{vol} / \mathrm{vol}])$ at a flow rate of $1.0 \mathrm{~mL} / \mathrm{min}$ and the product was UV-detected at $240 \mathrm{~nm}$ using an Agilent 1260 HPLC system.

\section{Transcriptional Assay by Real-time Polymerase Chain Reaction Analysis}

First, total RNA was isolated from the strains and used as the template to synthesize cDNA samples. Fermentation broths $(10 \mathrm{~mL})$ of MOX-101 and $\Delta$ nemR collected at day 4, 6, and 8 were centrifuged at $10,000 \times \mathrm{g}$ for 5 minutes, and pellets were ground into powder in liquid nitrogen. Next, an Ultrapure RNA Kit (CWBIO, China) was used for total RNA extraction according to the user manual provided by the manufacturer. The obtained RNA sample was treated with RNase-free DNase I (Takara, China) to remove the remaining gDNA. To obtain cDNA samples, reverse transcription(RT) was performed using M-MLV Reverse Transcriptase (Promega, United States).

Then, a cotranscriptional experiment utilizing RT-PCR was employed to investigate whether two nemadectin biosynthetic structural or regulatory genes spanning a short intergenic region and transcribed in the same direction were cotranscribed. Primers used to amplify the cDNA sample of MOX-101 were nemA1-2A2-F/R, nemA2C-F/R, nemA3E-F/R, and nemA4A3-F/R. gDNA and RNA samples without RT 
served as the positive and negative controls, respectively, in the PCR analyses.

Then, quantitative RT-PCR (qRT-PCR) was performed by TB Green $^{\mathrm{TM}}$ Premix Ex Taq ${ }^{\mathrm{TM}}$ II (Takara, China) according to the manufacturer's instructions. qRT-PCR experiments were performed to assay samples collected for 4,6 , and 8 days of fermentation. One gene was selected from each transcriptional unit for transcriptional analysis. Five primer pairs, including nemG-F/R, nemF-F/R, nemA1-2-F/R, nemA3-F/R, and nemA4$\mathrm{F} / \mathrm{R}$, were used in the qRT-PCR tests. Transcriptional levels of the tested genes were normalized using $h r d B$ (TU94_24720) as the internal control. ${ }^{19}$ Each qRT-PCR analysis was repeated three times, and the error bar represents the standard deviation.

\section{Results}

\section{NemR Functions as an Activator in the Biosynthesis of Nemadectin}

To study the function of nem $\mathrm{R}$ in nemadectin biosynthesis, we constructed the $\Delta$ nemR mutant strain with deletion of the nemR gene using MOX-101 as the parental strain (-Fig. 3A). Two strains were cultured in the fermentation medium, and broths were collected for 4,6 , and 8 days. The result showed that nemadectin production was significantly decreased in $\Delta n e m R$ with the deletion of the nemR gene. Nemadectin yield in $\Delta n e m R$ was decreased by approximately $80 \%$ in comparison with the parental strain MOX-101 ( - Fig. 3B). To further verify the function of NemR, we performed a complementation experiment in which the complemented plasmid pnemR was introduced into the $\Delta$ nemR mutant ( - Fig. 3A). In pnemR, $n e m \mathrm{R}$ was driven by the native promoter. The result showed that the complementation of nemR could restore the yield level of nemadectin to that of the parental strain (-Fig. 3C), revealing that the decline in nemadectin yield was mainly due to $n e m \mathrm{R}$ inactivation. These results demonstrated that NemR acts as an activator for nemadectin biosynthesis in S. cyaneogriseus.

\section{qRT-PCR Analysis of Transcriptional Levels of the Nemadectin Biosynthetic Gene Cluster}

A transcriptional assay was performed to further study the function of the NemR protein in nemadectin production. The gene cluster for nemadectin biosynthesis contains 10 structural genes and one regulatory gene ( $\mathbf{F i g}$. 4A). The result of the cotranscriptional experiment suggested that there were five cotranscriptional units altogether in the gene cluster of nemadectin biosynthesis, including nemG, nem $\mathrm{F}$, nem $\mathrm{A} 1-1-\mathrm{C}$, nemA3-D, and nemA4 (-Fig. 4B).

The transcriptional assay result indicated that nem $\mathrm{R}$ deletion strongly affects the transcription of nemadectin biosynthetic genes. As shown in - Fig. 4C, compared with the parental strain MOX-101, transcriptional levels of all nemadectin biosynthetic genes in the knockout strain $\Delta$ nemR were significantly decreased. Among these structural genes, transcriptional levels of nem $\mathrm{G} / \mathrm{F}$ and nemA1-1/A1-2/A2/C/D/E/A3/A4 in AnemR were only approximately $30 \%$ and less than $3 \%$ of those in MOX-101. These results suggested that nem $\mathrm{R}$ might play a positive regulatory role in nemadectin production by enhancing the expression of the nemadectin biosynthetic gene cluster.

A

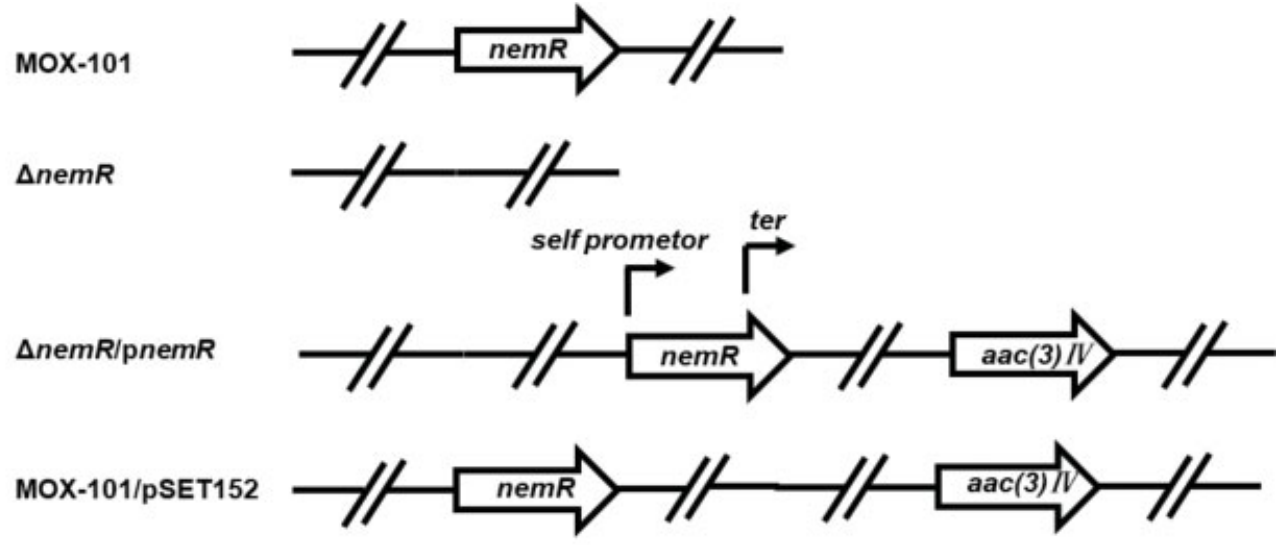

B

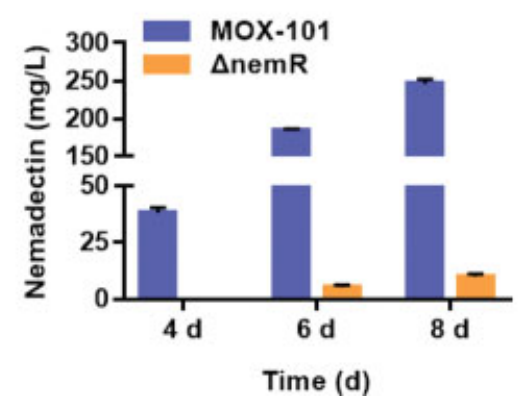

C

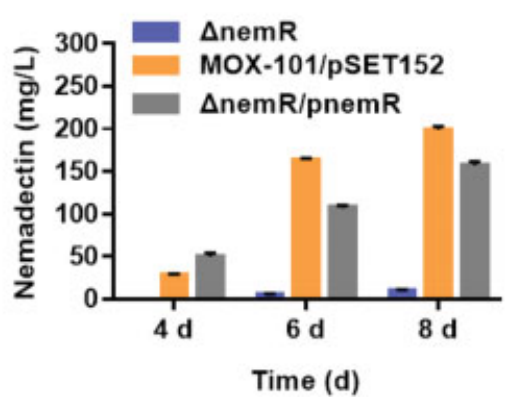

Fig. 3 Effect of nemR disruption on nemadectin production. (A) Genotypes of S. cyaneogriseus MOX-101 and its recombinant strains. (B) Nemadectin production in the parental strain MOX-101 and the mutant strain $\Delta$ nemR. (C) Nemadectin production in the mutant strains $\Delta$ nemR, MOX-101/pSET152, and $\Delta$ nemR/pnemR. 

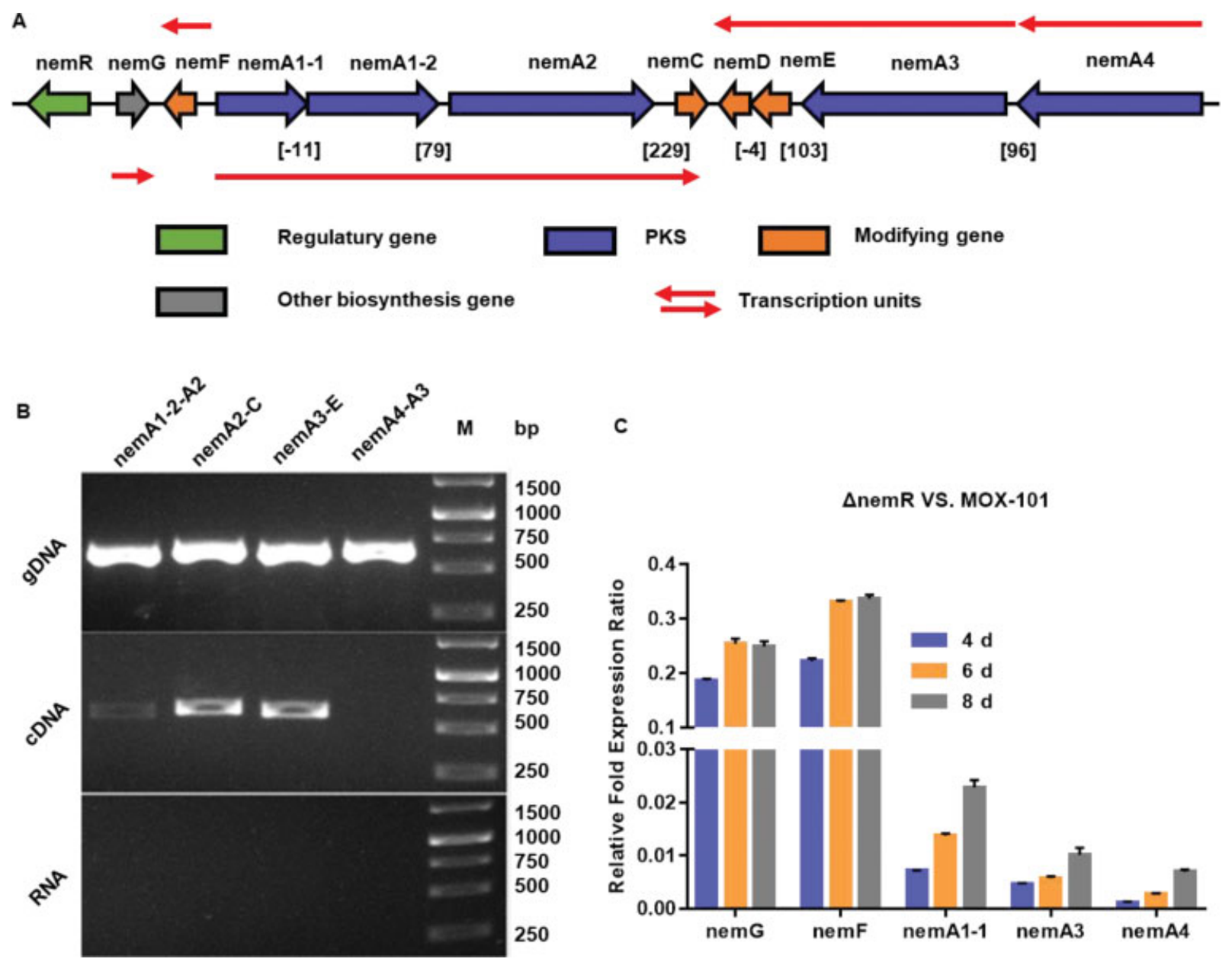

c

$\Delta$ nemR VS. MOX-101

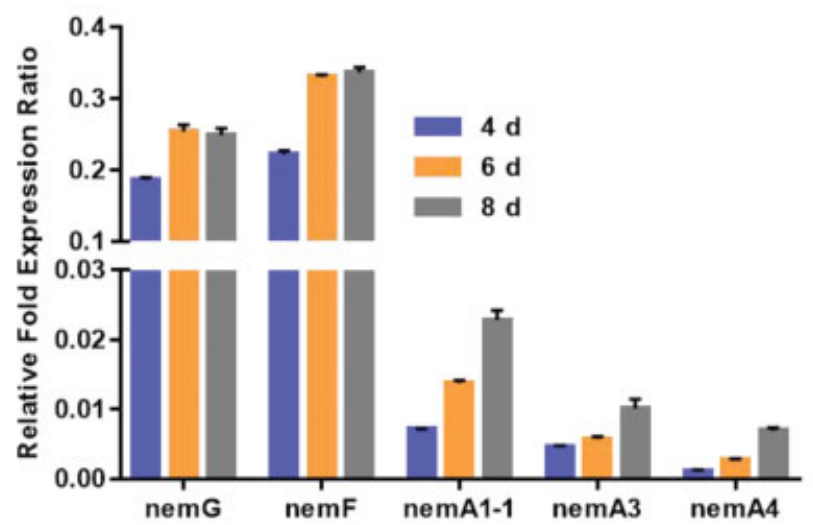

Fig. 4 Transcriptional analysis of the nemadectin biosynthetic genes upon nemR disruption in S. cyaneogriseus MOX-101. (A) Genetic map of the nemadectin biosynthetic gene cluster. (B) Cotranscriptional analysis of the nemadectin biosynthetic gene cluster. Genomic DNA (gDNA) and RNA samples without reverse transcription (RT) were used as the positive and negative controls, respectively. (C) Relative transcription levels between the parental strain MOX-101 and the knockout strain $\Delta n e m R$.

\section{Effect of Overexpression of nemR on Nemadectin Production}

To enhance nemadectin yield, pnemR under the control of the native promoter was transferred into MOX-101 to generate the overexpression strain MOX-101/pnemR. As a control, the control plasmid pSET152 was transferred into MOX-101 to generate MOX-101/pSET152. Three strains, MOX-101, MOX101/pnemR, and MOX-101/pSET152, were cultured for 4, 6, and 8 days, and broths were collected to detect the fermentation products by HPLC analysis. The result showed that compared with the original strain MOX-101, MOX-101/pnemR improved nemadectin production by $56.5 \%$, while the effect of MOX-101/pSET152 on nemadectin production was similar to that of MOX-101 ( $\mathbf{F i g . ~ 5 A ) . ~ T h e s e ~ r e s u l t s ~ s u g g e s t e d ~ t h a t ~ t h e ~}$ increase in production of MOX-101/pnemR was due to an extra copy of the gene nemR.

To confirm whether the improvement in nemadectin yield was caused by the improved expression of nemadectin biosynthetic genes, a transcriptional assay was performed by qRT-PCR analysis to determine the transcriptional levels of the genes in MOX-101 and MOX-101/pnemR. The result indicated that the transcription levels of the nemadectin biosynthetic genes in MOX-101/pnemR were higher than those in MOX-101
( - Fig. 5B). Among these genes, the transcription of nemG/F and nemA1-1/A3/A4 improved above 30 and $100 \%$, respectively.

To further increase the nemadectin yield, MOX-101/ pnemR-ermEp* was obtained by transforming pnemR-ermEp* into MOX-101, in which nemR is driven by the strong constitutive promoter ermEp*. As shown in - Fig. 5A, the production of MOX-101/pnemR-ermEp* was 10.9\% higher than that of MOX-101/pnemR and 73.5\% higher than that of MOX-101.

\section{Effect of Overexpression of Nemadectin Biosynthetic Gene Cluster on Nemadectin Production}

The result of the transcriptional assay in MOX-101 and MOX$101 /$ pnemR suggested that the improvement in nemadectin yield was caused by the increase in transcription levels of nemadectin biosynthetic genes. Then we attempted to directly overexpress the nemadectin biosynthetic gene cluster to increase its yield.

In the early stage of the experiment, several attempts were made to clone a complete nemadectin biosynthetic gene cluster, but all failed because of its large size $(\sim 90.9 \mathrm{~kb})$. Therefore, two modules, MOX1 (50 kb) and MOX2 (41.7 kb), of the complete biosynthetic gene cluster were cloned respectively, and then the two modules were spliced together to 
A
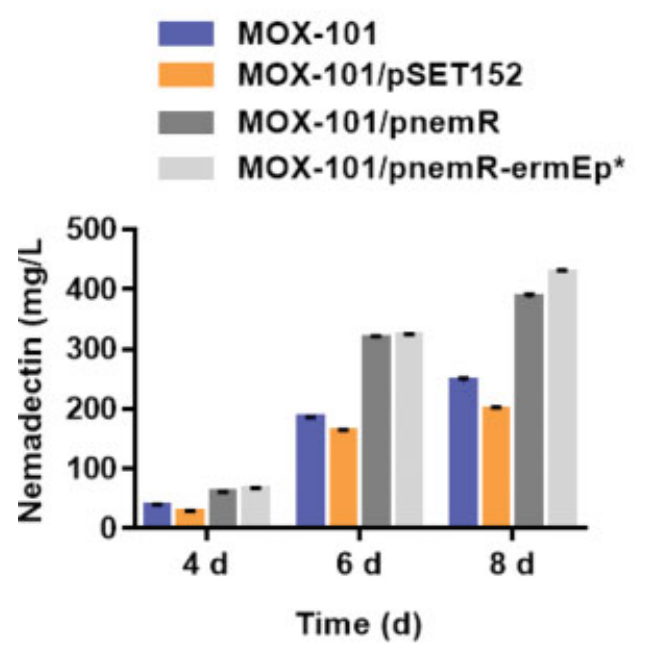

\section{B MOX-101/pnemR VS. MOX-101}

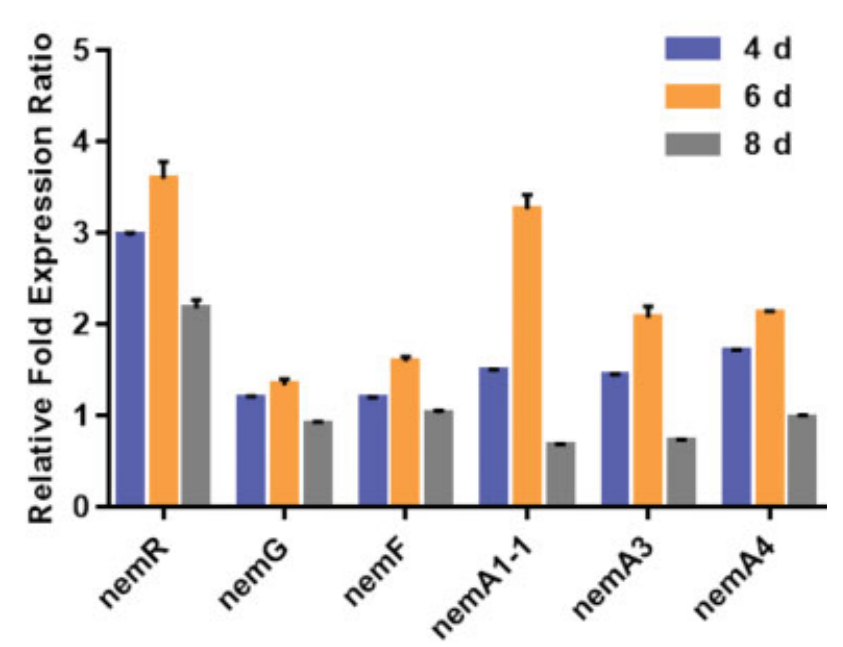

Fig. 5 Effect of nemR overexpression on nemadectin production. (A) Nemadectin production in MOX-101, MOX-101/pSET152, MOX-101/pnemR, and MOX101/pnemR-ermEp*. (B) Relative transcription levels between the parental strain MOX-101 and the overexpression strain MOX-101/pnemR.
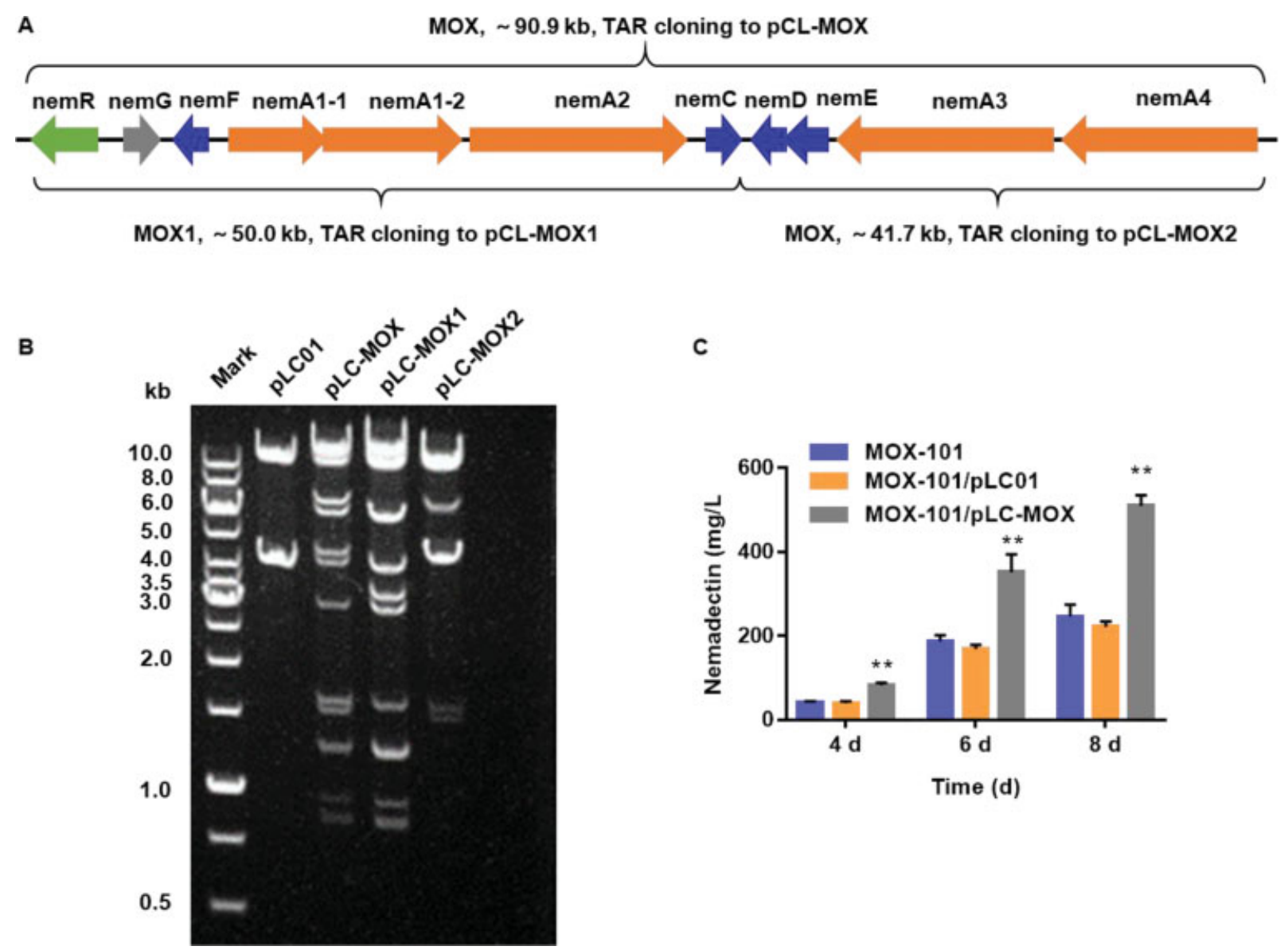

Fig. 6 Effect of biosynthetic gene cluster overexpression on nemadectin production. (A) Map of captured nemadectin biosynthetic gene cluster by the CRISPR-TAR method. (B) Restriction analysis of plasmids pCL01, pCL-MOX, pCL-MOX1, and pCL-MOX2. (C) Nemadectin production in MOX101, MOX-101/pCL01, and MOX-101/pCL-MOX.

obtain the complete nemadectin biosynthetic gene cluster MOX (-Fig. 6A). Plasmids pCL01, pCL-MOX1, pCL-MOX2, and PCL-MOX were verified by the restriction enzyme Kpnl digestion, which showed that gene clusters MOX1, MOX2, and MOX were successfully cloned ( $\mathbf{- F i g . 6 B}$ ). The plasmid pCL-
MOX, containing the complete nemadectin biosynthetic gene cluster, was transferred into MOX-101 to generate the overexpression strain MOX-101/pCL-MOX. As a control, the control plasmid pCL01 was transferred into MOX-101 to generate MOX-101/pCL01. Three strains, MOX-101, MOX-101/pCL01, 
and MOX-101/pCL-MOX, were cultured for 4,6 , and 8 days. The result showed that MOX-101/pCL-MOX significantly increased the production of nemadectin by $108.6 \%(509 \mathrm{mg} / \mathrm{L})$ compared with the MOX-101 (-Fig. 6C).

\section{Discussion}

Strain improvement is very important for the industrialization of microbial medicine. Previously, random selection, such as UV mutagenesis and atmospheric and room temperature plasma mutation, and rational breeding were used to increase nemadectin yield. ${ }^{11,20}$

Overexpression of transcriptional regulators is an effective and commonly used method to improve antibiotic production, especially when regulators were driven by strong constitutive promoters, such as ermEp*.21-23 In 2019, a positive transcriptional regulator, NemR, involved in nemadectin biosynthesis was reported. ${ }^{15}$ In this study, we overexpressed nemR in MOX101 under the control of both native and strong constitutive promoters. The result showed that the yield of nemadectin was considerably improved compared with that of MOX-101 owing to the increase in transcription levels of nemadectin biosynthetic genes. Therefore, we attempted to directly overexpress the nemadectin biosynthetic gene cluster to increase its yield. This is the first report that an extra copy of the complete nemadectin biosynthesis gene cluster was introduced and overexpressed in a nemadectin-producing strain and the production was more than doubled.

Overexpression of nem $\mathrm{R}$ or nemadectin biosynthesis gene cluster increased the transcriptional level of nemadectin biosynthesis-related genes and thus improved nemadectin production. This revealed that the low fermentation yield of nemadectin may be due to the low transcriptional level of nemadectin biosynthesis-related genes. These findings helped us to elucidate nemadectin biosynthesis, and provide approaches to enhance nemadectin production by modifying new positive regulatory genes or further increasing the copy number of the nemadectin biosynthetic gene cluster in MOX101/pCL-MOX.

\section{Funding}

This work was supported by the National Key Research and Development Program (Grant No. 2019YFA0905400) and the Program of Shanghai Technology Research Leader (Grant No. 19XD1433200).

\section{Conflict of Interest}

The authors declare that they have no conflict of interest.

\section{References}

1 Carter GT, Nietsche JA, Hertz MR, et al. LL-F28249 antibiotic complex: a new family of antiparasitic macrocyclic lactones. Isolation, characterization and structures of LL-F28249 alpha, beta, gamma, lambda. J Antibiot (Tokyo) 1988;41(4):519-529

2 Doscher ME, Wood IB, Pankavich JA, Ricks CA. Efficacy of nemadectin, a new broad-spectrum endectocide, against natural infections of canine gastrointestinal helminths. Vet Parasitol 1989;34(3):255-259

3 Mishima H, Kurabayashi M, Tamura C, et al. The structures of milbemycin $\alpha 1,2,3,4,5,6,7,8,9,10$, and $\beta 1$. Paper presented at: Symposium on the Chemistry of Natural Products, symposium papers; 1974

4 Burg RW, Miller BM, Baker EE, et al. Avermectins, new family of potent anthelmintic agents: producing organism and fermentation. Antimicrob Agents Chemother 1979;15(3):361-367

5 Sun Y, Zhou X, Tu G, Deng Z. Identification of a gene cluster encoding meilingmycin biosynthesis among multiple polyketide synthase contigs isolated from Streptomyces nanchangensis NS3226. Arch Microbiol 2003;180(2):101-107

6 Asato G, France DJ. 23-Imino Derivatives of LL-F28249 Compounds. U.S. Patent No. 4916154. April, 1990

7 Lanusse C, Lifschitz A, Virkel G, et al. Comparative plasma disposition kinetics of ivermectin, moxidectin and doramectin in cattle. J Vet Pharmacol Ther 1997;20(2):91-99

8 Prichard R, Ménez C, Lespine A. Moxidectin and the avermectins: consanguinity but not identity. Int J Parasitol Drugs Drug Resist 2012;2(2):134-153

9 Shoop WL, Haines HW, Michael BF, Eary CH. Mutual resistance to avermectins and milbemycins: oral activity of ivermectin and moxidectin against ivermectin-resistant and susceptible nematodes. Vet Rec 1993;133(18):445-447

10 Mounsey KE, Walton SF, Innes A, Cash-Deans S, McCarthy JS. In vitro efficacy of moxidectin versus ivermectin against Sarcoptes scabiei. Antimicrob Agents Chemother 2017;61(8):e00381-e17

11 Wang Z. Improvement of Agricultural Antibiotic Nemadectin Producing Strain by Rational Screening [in Chinese]. Heilongjiang: Northeast Agricultural University; 2009

12 Zhang SH, Ruan QC, Wen B, et al. Preliminary study on fermentation of nemadectin, the agro-antibiotic against parasites. Zhongguo Nongxue Tongbao 2014;30(21):228-234

13 Li M, Yang LF. A Method to Improve the Fermentation Yield of Nemadectin. China Patent No. CN104450823A. Mar, 2015

14 Tsou HR, Ahmed ZH, Fiala RR, et al. Biosynthetic origin of the carbon skeleton and oxygen atoms of the LL-F28249 alpha, a potent antiparasitic macrolide. J Antibiot (Tokyo) 1989;42(3):398-406

$15 \mathrm{Li} \mathrm{C}$, He H, Wang J, et al. Characterization of a LAL-type regulator NemR in nemadectin biosynthesis and its application for increasing nemadectin production in Streptomyces cyaneogriseus. Sci China Life Sci 2019;62(3):394-405

16 Huang H, Zheng G, Jiang W, Hu H, Lu Y. One-step high-efficiency CRISPR/Cas9-mediated genome editing in Streptomyces. Acta Biochim Biophys Sin (Shanghai) 2015;47(4):231-243

17 Li L, Wei K, Liu X, et al. aMSGE: advanced multiplex site-specific genome engineering with orthogonal modular recombinases in actinomycetes. Metab Eng 2019;52:153-167

18 Lee NC, Larionov V, Kouprina N. Highly efficient CRISPR/Cas9mediated TAR cloning of genes and chromosomal loci from complex genomes in yeast. Nucleic Acids Res 2015;43(8):e55

19 Livak KJ, Schmittgen TD. Analysis of relative gene expression data using real-time quantitative PCR and the 2(-Delta Delta C(T)) method. Methods 2001;25(4):402-408

20 Liu RH, Ren QW, Chen RR, et al. Application of ARTP mutation technology in breeding of nemadectin strain. Guangzhou Huagong 2018;(13):122-124

21 Bibb MJ. Regulation of secondary metabolism in streptomycetes. Curr Opin Microbiol 2005;8(2):208-215

22 Wang W, Li X, Wang J, Xiang S, Feng X, Yang K. An engineered strong promoter for streptomycetes. Appl Environ Microbiol 2013;79(14):4484-4492

23 Bibb MJ, Janssen GR, Ward JM. Cloning and analysis of the promoter region of the erythromycin resistance gene (ermE) of Streptomyces erythraeus. Gene 1985;38(1-3):215-226 